Notfall Rettungsmed

https://doi.org/10.1007/s10049-021-00929-2

Angenommen: 9. Juni 2021

๑ Der/die Autor(en) 2021, korrigierte Publikation 2022

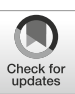

\section{Neurologisches Ergebnis und allgemeiner Gesundheitszustand bei Langzeitüberleben nach außerklinischer kardiopulmonaler Reanimation}

\author{
J. M. Poth ${ }^{1}$. C. M. Buschmann ${ }^{2}$. J. Kappler ${ }^{1}$ - U. Heister' ${ }^{1}$ S. F. Ehrentraut ${ }^{1}$. \\ S. Muenster' · C. J. Diepenseifen ${ }^{3} \cdot$ R. Ellerkmann ${ }^{4} \cdot$ J.-C. Schewe' \\ 'Klinik für Anästhesiologie und Operative Intensivmedizin, Universitätsklinikum Bonn, Venusberg- \\ Campus 1, Bonn, Deutschland \\ ${ }^{2}$ Abteilung für Anästhesie, Intensiv- und Schmerzmedizin, Marienhospital Brühl GmbH, Brühl, \\ Deutschland \\ ${ }^{3}$ Rettungsdienst Rhein-Sieg-Kreis, Der Landrat, Siegburg, Deutschland \\ ${ }^{4}$ Klinik für Anästhesiologie, operative Intensivmedizin und postoperative Schmerztherapie, Klinikum \\ Dortmund gGmbH, Dortmund, Deutschland
}

\section{Zusatzmaterial online}

Die Online-Version dieses Beitrags (https://doi. org/10.1007/s10049-021-00929-2) enthält eine weitere Tabelle zum Ergebnis der Befragung zur Einschätzung des Gesundheitszustandes der Langzeitüberlebenden (Suppl. Tabelle 1)

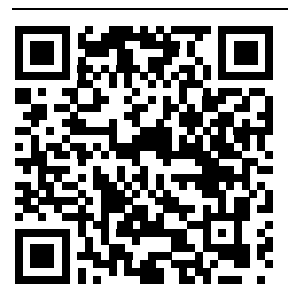

QR-Code scannen \& Beitrag online lesen
Die Inzidenz begonnener Reanimationen nach einem außerklinischen Herz-Kreislauf-Stillstand (,out-of-hospital cardiac arrest" [OHCA]) liegt in Europa bei 56-66 pro 100.000 Einwohner und Jahr [10, 11]. Studien zeigen OHCA-Überlebensraten bis zur Krankenhausentlassung von lediglich 7,9 bis $13,2 \%$ [7, 9, 13, 26]. Im Verlauf versterben zwei Drittel der nach OHCA zunächst ins Krankenhaus aufgenommenen Patienten, vorrangig an einem neurologischen Schaden [17, 18]. Insgesamt werden in Deutschland 9\% der prähospital reanimierten Patienten in einem guten neurologischen Zustand entlassen [9]. Im Gegensatz zu anderen Rettungsdienstsystemen [2, 26] liegen für Deutschland keine publizierten Daten zum weiteren Verlauf nach Entlassung und dem neurologischen Langzeitüberleben vor. Erkenntnisse und Einblicke dahingehend sind jedoch auch aus Gründen der Ressourcenplanung, ethischen Gesichtspunkten und gesundheitspolitischen Versorgungsaspekten von großer Bedeutung. Für den individuellen Patienten ist das Erreichen des bestmöglichen Behandlungsergebnisses nach Reanimation, nämlich ein Gesundheitszustand ohne kognitive Beeinträchtigung bei akzeptabler Lebensqualität, entscheidend. Dafür bedarf es neben den primären notärztlichen Bemühungen einer suffizienten und durchgehenden Versorgungskette im Krankenhaus. Der relevanteste Ergebnisparameter dieser Anstrengungen ist vorrangig das neurologische Langzeitergebnis $[2,20]$.

Die vorliegende Studie untersucht das Überleben, die neurologische Beeinträchtigung und Lebensqualität aller Patienten im Rettungsdienst der Bundesstadt Bonn 2,5-5 Jahre nach OHCA. Auf Grundlage der erhobenen Daten wird zudem untersucht, ob und welche Zusammenhänge zwischen den erfassten Variablen und dem neurologischen Langzeitergebnis bestehen (Kurzzusammenfassung siehe - Abb. 1).

\section{Material und Methoden}

\section{Studiendesign und Datenerhebung}

Es handelt sich um eine retrospektive Analyse aller durch den Rettungsdienst der Bundesstadt Bonn begonnenen Reanimationen im Zeitraum vom 01.01.2011 bis zum 30.06.2013 und Befragung der Langzeitüberlebenden. Patienten jünger als 18 Jahre wurden ausgeschlossen. Das vorliegende Studiendesign wurde durch die Ethikkommission der Medizinischen 
Fakultät der Rheinischen Friedrich-Wilhelms-Universität Bonn genehmigt (Lfd. Nr. 034/17).

Grundlage der Untersuchung waren die Notarzteinsatzprotokolle und das Deutsche Reanimationsregister. Das 30-TageÜberleben sowie die Verlegung oder Entlassung aus dem Krankenhaus wurden den Routineaufzeichnungen der aufnehmenden Krankenhäuser entnommen. Im Juli 2015 wurde auf Grundlage der Daten der Einwohnermeldeämter das weitere Überleben dieser Patienten ermittelt. Zur weiteren Erfassung des Langzeitüberlebens und neurologischen Ergebnisses sowie zur Selbsteinschätzung des Gesundheitszustands wurden die tatsächlich noch lebend gemeldeten Patienten im Februar 2016 angeschrieben und gebeten, einen Fragebogen mit 15 Fragen in Anlehnung an den etablierten und validierten SF-12 $[2,19,29]$ zu beantworten (SupplementTab. 1, siehe Hinweis am Anfang des Artikels). Auf Grundlage der Antworten wurde das neurologische Ergebnis in eine Cerebral Performance Category (CPC) klassifiziert [15, 30]. CPC-1 („good cerebral performance") und CPC-2 (moderate Einschränkungen, aber unabhängig von anderen) wurden als gutes neurologisches Ergebnis definiert und als CPC $\leq 2$ zusammengefasst. Das selbstständige Ausfüllen des Fragebogens wurde auf dem Bogen erfragt und als Indikator einer $\mathrm{CPC} \leq 2$ eingestuft. Tod (CPC-5), vegetativer Status (CPC-4) und Einschränkungen mit permanenter Abhängigkeit von anderen (CPC3) wurden als schlechtes neurologisches Ergebnis (CPC $\geq 3)$ definiert.

\section{Abkürzungen}

\begin{tabular}{ll} 
CPC & Cerebral Performance Category \\
CPR & "Cardiopulmonary resuscitation" \\
DGAl & $\begin{array}{l}\text { Deutsche Gesellschaft für Anästhe- } \\
\text { siologie und Intensivmedizin }\end{array}$ \\
NEF & Notarzteinsatzfahrzeug \\
OHCA & "Out-of-hospital cardiac arrest" \\
PEA & Pulslose elektrische Aktivität \\
ROSC & "Return of spontaneous circulation" \\
ROSC-KH & Krankenhausaufnahme mit \\
& Spontankreislauf \\
RTW & Rettungswagen \\
$V F$ & Ventrikuläres Flimmern \\
$V T$ & Ventrikuläre Tachykardie \\
\hline
\end{tabular}

Fragestellung: Für Deutschland liegen nur wenige Daten zum Gesundheitszustand und Langzeitüberleben nach außerklinischer Reanimation vor. Die vorliegende Studie untersucht den allgemeinen Gesundheitszustand und das neurologische Langzeitergebnis 2,5 bis 5 Jahre nach dem Reanimationsereignis sowie den Zusammenhang zwischen Behandlungsergebnis und präklinischen Patienten- und Behandlungsfaktoren.

Methodik: Alle über einen Zeitraum von 30 Monaten (2011-2013) durch den Rettungsdienst der Stadt Bonn durchgeführten Reanimationen wurden auf Grundlage der Notarzteinsatzprotokolle und des Deutschen Reanimationsregisters retrospektiv analysiert. Der allgemeine Gesundheitszustand wurde in Anlehnung an den SF-12 erfasst und das neurologische Langzeitergebnis kategorisiert (Cerebral Performance Category $[\mathrm{CPC}]$ ). Die vorliegenden Daten wurden einer uni- und multivariaten logistischen Regressionsanalyse unterzogen.

Ergebnis: Von insgesamt 458 Patienten überlebten 17,9\% bis zur Krankenhausentlassung, $13,8 \%$ mehr als 2,5 Jahre und 7,7\% bis zum Stichtag der Befragung. Von den noch lebenden Patienten hatten 85,3\% ein gutes neurologisches Ergebnis (CPC $\leq 2)$, welches durch ein geringeres Alter, einen beobachteten Kollaps, die Durchführung einer Defibrillation und das Ausbleiben einer Vasopressorgabe begünstigt wurde (multivariate Analyse). 74,2\% der Überlebenden beschrieben ihren Gesundheitszustand als gut.

Interpretation: Insgesamt überleben langfristig nur wenige Patienten einen außerklinischen Herz-Kreislauf-Stillstand. Die überlebenden Patienten beurteilen ihren Gesundheitszustand als gut und zeigen ein gutes neurologisches Ergebnis. Dabei hängt dieses Behandlungsergebnis von denselben Parametern wie der primäre Reanimationserfolg (Überleben bis Krankenhausaufnahme) ab. Die Etablierung klinischer Instrumente zur frühen Prognoseerstellung ist wünschenswert. Hierzu sind weitere Langzeituntersuchungen größerer Patientenkollektive mit Zugriff auf Routinedaten notwendig.

\section{Schlüsselwörter}

Herz-Kreislauf-Stillstand · Herzstillstand · Langzeitoutcome $\cdot$ Reanimation

\section{Fehlende Daten}

Das Fehlen der Ergebnisdaten der nicht nachverfolgbaren Patienten wurde als nicht zufällig beurteilt (statistisch: „missing not at random"). Die Auswertungen des Langzeitüberlebens erfolgten unter Annahme des besten (Annahme: fehlende Patienten leben) und schlechtesten (Annahme: fehlende Patienten verstorben) Szenarios sowie unter Ausschluss fehlender Daten. Für die weiteren Regressionsanalysen wurden fehlende Ergebnisdaten als CPC $\geq 3$ klassifiziert (schlechtestes Szenario).

\section{Struktur des Rettungsdiensts der Stadt Bonn}

Der Rettungsdienst der Bundesstadt Bonn versorgte im Untersuchungszeitraum ca. 320.000 Einwohner auf $141 \mathrm{~km}^{2}$ Fläche sowie Teile der Nachbarkommunen mit drei Notarzteinsatzfahrzeugen und 14 Rettungswagen im Rendezvous- system. Im Untersuchungszeitraum waren weder ein First-responder-System noch ein System zur telefonischen Anleitung der Laienreanimation etabliert. Alle Reanimationen wurden entsprechend den gültigen Leitlinien (Reanimationsleitlinien $2010[6,21])$ durchgeführt. Der Rettungsdienst war über die entsprechenden Vorschriften hinaus mit Larynxmasken und -tuben, einem Gerät zur mechanischen Thoraxkompression sowie Coolpacks zur Einleitung einer Hypothermiebehandlung ausgestattet. Überlebende Patienten wurden verschiedenen Krankenhäusern zugewiesen, abhängig von Verfügbarkeit und Fachabteilung (z.B. Herzkatheterlabor).

\section{Regressionsanalyse und Statistik}

Alle statistischen Berechnungen wurden in SPSS Statistics 21 (IBM) durchgeführt.

Es wurde das neurologische Ergebnis in zwei Patientenpopulationen betrachtet: 1) Alle begonnenen Reanimationen 


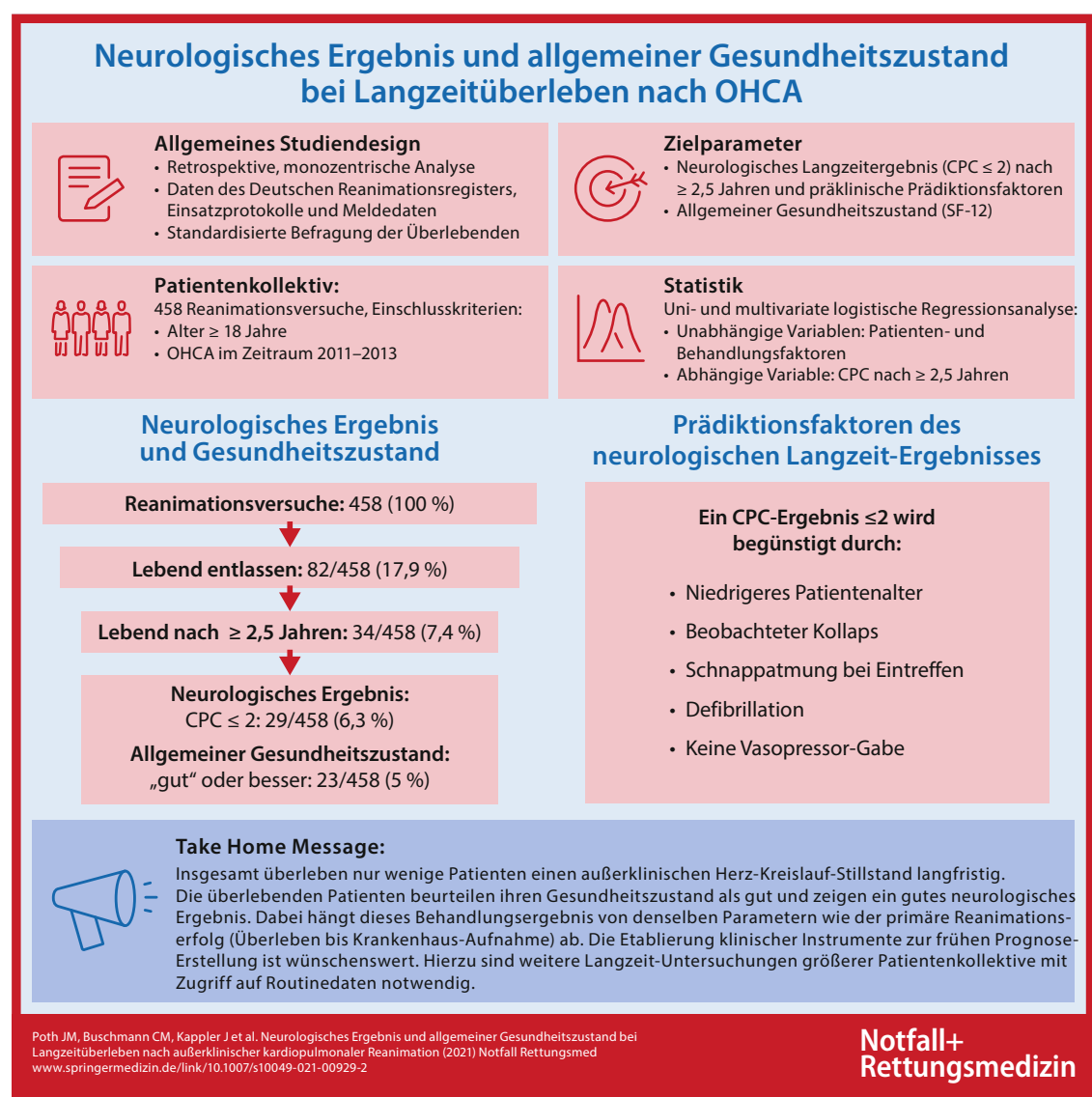

Abb. 1 ॥ Visual Abstract - Kurzzusammenfassung des Studiendesigns und der wesentlichen Ergebnisse

("rgesamt") und 2) alle Patienten, die mit ROSC im Krankenhaus aufgenommen worden waren („,ROSC-KH“). Zur Untersuchung eines Zusammenhangs zwischen einem $C P C \leq 2$-Ergebnis und patientenund behandlungsabhängigen Faktoren wurden im Reanimationsregister erfasste Parameter als unabhängige Variablen einer univariaten logistischen Regressionsanalyse (binär) unterzogen, mit einem Langzeit-CPC $\leq 2$-Ergebnis als abhängige Variable. Kategoriale Variablen wurden zur einfacheren Betrachtung teilweise, wie im Text und in den Tabellen angegeben, transformiert. Eine Beeinflussung der Wahrscheinlichkeit eines $\mathrm{CPC} \leq 2$ Ergebnisses durch eine unabhängige Variable wurde angenommen, wenn $p<0,05$ für den $\times 2$-Test des Modells und wenn das Konfidenzintervall $(95 \%-\mathrm{Cl})$ der Odds Ratio (OR) den Wert 1 nicht kreuzte.

Für die weitere Analyse wurden nur die Variablen berücksichtigt, für die in der univariaten Analyse ein Zusammenhang gezeigt worden war. Mittels sequenziel- führt. Dies entspricht einer Inzidenz von 57/100.000/Jahr.

- Abb. 2 zeigt die Überlebensraten der Patienten zu unterschiedlichen Zeitpunkten im Behandlungsverlauf. Dabei wurden $17,9 \%$ (82/458) der Patienten lebend aus dem Krankenhaus entlassen und 13,8\% (63/458) überlebten länger als 2 Jahre.

Am Stichtag der Befragung waren zehn weitere Patienten verstorben, bei drei Patienten konnten keine Meldedaten abgefragt werden, vier Patienten waren unbekannt verzogen. Ein Fragebogen wurde nicht beantwortet, elf Fragebögen gar nicht zurückgeschickt. Somit konnten insgesamt 34 Patienten nachverfolgt werden. Ein Patient wollte nicht an der Befragung teilnehmen. Zum Stichtag ergibt sich eine Überlebensrate von 7,7\% (34/439; Ausschluss nicht verfolgbarer Patienten) bzw. von 7,4\% (34/458; Annahme: nicht verfolgbare Patienten verstorben) bis $11,6 \%$ (53/458; Annahme: nicht verfolgbare Patienten leben).

\section{Neurologisches Langzeitergebnis und prognostisch relevante Faktoren}

Bei 4,4\% (20/458) aller Patienten konnte keine CPC ermitteltwerden (• Abb. 2: „Fehler Meldedaten“, „Keine Antwort”, „Befragung nicht verwertbar"). Ein Patient wurde der CPC-3, drei Patienten der CPC-4 und 29 Patienten der $\mathrm{CPC} \leq 2$ zugeordnet. Somit erreichten 6,3\% (29/458; Annahme: nicht verfolgbare Patienten $\mathrm{CPC} \geq 3$ ) bis 10,7\% (49/458; Annahme: nicht verfolgbare Patienten $\mathrm{CPC} \leq 2$ ) ein gutes neurologisches Ergebnis. Nach Ausschluss der nicht verfolgbaren Patienten war dies bei $6,6 \%$ der Fall (29/438). Fehlende Ergebnisse wurden als $C P C \geq 3$ eingestuft und als solche in den folgenden Regressionsanalysen berücksichtigt (s. Diskussion).

Zur weiteren Auswertung wurden die erfassten Parameter zum Teil gruppiert (z. B.:Zusammenfassen der EKG-Rhythmen PEA und Asystolie als nicht schockbare Rhythmen). Dies wurde im Gesamtkollektiv (• Tab. 1) und im Kollektiv der mit ROSC aufgenommenen Patienten durchgeführt (- Tab. 2).

Im Untersuchungszeitraum von 30 Monaten wurden 458 Reanimationen durch den Rettungsdienst der Stadt Bonn durchge-
Univariate logistische Regression. Im Gesamtkollektiv (- Tab. 1) waren die folgenden Parameter mit einer geringeren 


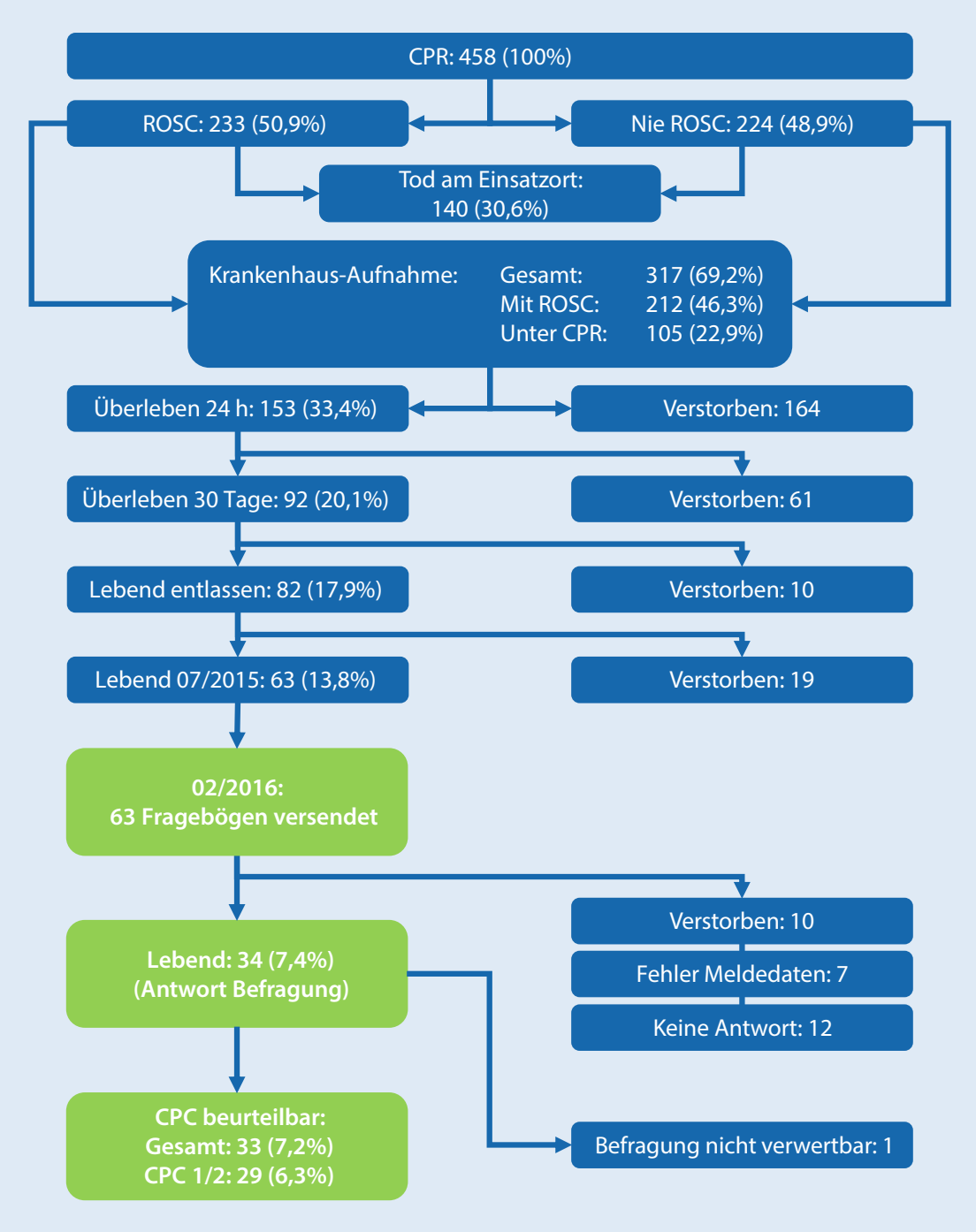

Abb. $2 \Delta$ Darstellung des Reanimationsoutcomes und Nachverfolgung der Patienten zum langfristigen Behandlungsergebnis

Wahrscheinlichkeit für ein gutes neurologisches Langzeitergebnis ( $C P C \leq 2)$ assoziiert: höheres Patientenalter, Reanimation in einer Wohnung, nicht schockbare initiale EKG-Rhythmen, Fehlen von Schnappatmung, vermutete nichtkardiale Ursache, nicht beobachteter Kollaps, keine Laienreanimation, keine Defibrillation, Vasopressorgabe, Zeitpunkt des ersten ROSC in Anwesenheit des Notarztes (im Vergleich zum Ersthelfer) und Krankenhausaufnahme unter laufender Reanimation. Des Weiteren war ein gutes neurologisches Langzeitergebnis wahrscheinlicher bei Reanimation in einer Arztpraxis oder im öffentlichen Raum (in Anlehnung an die Utstein-Kriterien [22]) sowie bei wiedererlangtem Bewusstsein oder notwendiger Analgosedierung zum Zeitpunkt der Krankenhausaufnahme. Alle überlebenden Patienten, die initial durch Ersthelfer reanimiert worden waren, zeigten ein gutes neurologisches Langzeitergebnis. Dieses Ergebnis erreichte kein Patient, der in einer Altenpflegeeinrichtung reanimiert worden war.

Die für das Gesamtkollektiv gezeigten Zusammenhänge konnten auch im ROSCKH-Kollektiv nachgewiesen werden, mit den Ausnahmen des initialen Atemmusters und des Zeitpunkts des ersten ROSC, für die kein statistischer Zusammenhang gezeigt werden konnte.

Multivariate logistische Regression. Wir untersuchten, ob ein gutes neurologisches
Langzeitergebnis im Gesamtkollektiv auf Grundlage der unter Reanimation erhobenen Daten, also unter Ausschluss der Ergebnisvariablen "Jemals ROSC", "1. ROSC", "Krankenhausaufnahme" und „Bewusstseinslage bei Übergabe" vorhersagbar ist. Dazu berücksichtigten wir alle verbleibenden Parameter, von denen ein gutes neurologisches Langzeitergebnis gemäß der univariaten Analyse abhängig war. Zur Vereinfachung wurden diese verbleibenden Daten in dichotome Variablen transformiert (z. B.: VF/VT - ja/ nein). Die multivariate logistische Regressionsanalyse zeigte, dass ein geringeres Alter, ein beobachteter Kollaps, die Durchführung einer Defibrillation während der Reanimation sowie das Ausbleiben einer Vasopressorgabe ein gutes neurologisches Langzeitergebnis begünstigten (AUC der ROC-Kurve 0,87). Das Vorliegen von Schnappatmung erhöhte die Wahrscheinlichkeit eines $C P C \leq 2$-Ergebnisses nicht signifikant, verbesserte aber das Gesamtmodell (AUC 0,90; - Tab. 3).

Das Langzeitüberleben (und damit die CPC) eines Patienten nach OHCA hängt auch von der Restlebenserwartung dieses Patienten vor der Reanimation ab. Nach Ausschluss der Variablen "Alter" wurde ein gutes neurologisches Langzeitergebnis durch einen beobachteten Kollaps, die Durchführung einer Defibrillation während der Reanimation, das Ausbleiben einer Vasopressorgabe und durch das Vorliegen von Schnappatmung begünstigt (AUC der ROC-Kurve 0,87; - Tab.3, kursiv).

Im ROSC-KH-Kollektiv konnte ein gutes neurologisches Langzeitergebnis anhand derselben Variablen "Alter", „Kollaps beobachtet", "Defibrillation" und "Vasopressorgabe“ prädiziert werden (AUC der ROC-Kurve: 0,83; - Tab. 4), die zusätzliche Berücksichtigung des Vorliegens von Schnappatmung bei Eintreffen des Rettungsdiensts verbesserte das Modell geringfügig (AUC der ROC-Kurve 0,87). Das Hinzufügen weiterer Parameter hatte keinen positiven Effekt auf die Testgenauigkeit. Auch unter Ausschluss des Parameters "Alter" war das neurologische Langzeitüberleben anhand der übrigen genannten Variablen prognostizierbar (AUC der ROC-Kurve 0,84), allerdings war die Spezifität dann deutlich schlechter. 


\begin{tabular}{|c|c|c|c|c|c|c|c|}
\hline Parameter & & $\begin{array}{l}\text { Gesamt } \\
(\%)^{\mathrm{a}}\end{array}$ & $\begin{array}{l}\mathrm{CPC} \leq 2 \\
(\%)^{\mathrm{b}}\end{array}$ & OR & $\mathrm{Cl}(95 \%)$ & $\begin{array}{l}X^{2} \text { (Mo- } \\
\text { dell) }\end{array}$ & $p\left(X^{2}\right)$ \\
\hline $\begin{array}{l}\text { Alter (in } \\
\text { Jahren) }\end{array}$ & - & $\emptyset 69,2$ & $\varnothing 57,9$ & 0,961 & $0,94-0,98$ & 13,30 & $<0,01$ \\
\hline \multirow[t]{2}{*}{ Geschlecht } & Weiblich & $151(33)$ & $7(4,6)$ & - & - & - & - \\
\hline & Männlich & $307(67)$ & $22(7,2)$ & - & - & - & - \\
\hline \multirow{13}{*}{$\begin{array}{l}\text { Einsatzort } \\
\text { (n/a: } 3 \\
[0,7 \%])\end{array}$} & Wohnung (1) & $267(58,3)$ & $10(3,7)$ & $\begin{array}{l}0,25 \text { (vs. öffentlich } \\
\text { komb.) }\end{array}$ & $0,11-0,57$ & 22,23 & $<0,01$ \\
\hline & Altenpflegeeinrichtung (2) & $52(11,4)$ & $0(0)$ & - & - & - & - \\
\hline & Arbeitsplatz (3) & $10(2,2)$ & $3(30,0)$ & - & - & - & - \\
\hline & Arztpraxis (4) & $6(1,3)$ & $2(33,3)$ & 12,85 (vs. Wohnung) & $2,10-78,61$ & 22,23 & $<0,01$ \\
\hline & Straße (5) & $53(11,6)$ & $6(11,3)$ & - & - & - & - \\
\hline & Öffentlicher Raum (6) & $33(7,2)$ & $3(9,1)$ & - & - & - & - \\
\hline & Krankenhaus (7) & $17(3,7)$ & $2(11,8)$ & - & - & - & - \\
\hline & Massenveranst. (8) & $1(0,2)$ & $0(0)$ & - & - & - & - \\
\hline & Sonstiges (9) & $11(2,4)$ & $1(9,1)$ & - & - & - & - \\
\hline & Bildungseinrichtung (10) & $1(0,2)$ & $0(0)$ & - & - & - & - \\
\hline & Sportstätte (11) & $4(0,9)$ & $2(50,0)$ & - & - & - & - \\
\hline & Öffentl. kombiniert I $(3,5,6,8,10,11)$ & $102(22,3)$ & $12(13,7)$ & 4,09 (vs. Wohnung) & $1,75-9,54$ & 22,23 & $<0,01$ \\
\hline & Öffentl. kombiniert II $(3,4,5,6,8,10,11)$ & $108(23,6)$ & $16(14,8)$ & 4,47 (vs. Rest) & $2,07-9,63$ & 14,28 & $<0,01$ \\
\hline \multirow{5}{*}{$\begin{array}{l}\text { Initialer } \\
\text { Rhythmus }\end{array}$} & \multirow[t]{2}{*}{ VF/VT } & \multirow[t]{2}{*}{$140(30,6)$} & \multirow[t]{2}{*}{$21(15,0)$} & 8,12 (vs. Asystolie) & $2,72-24,24$ & 23,38 & $<0,01$ \\
\hline & & & & 6,84 (vs. nicht schockbar) & $2,95-15,86$ & 23,10 & $<0,01$ \\
\hline & PEA & $130(28,4)$ & $4(3,1)$ & 0,18 (vs. VF/VT) & $0,06-0,54$ & 23,38 & $<0,01$ \\
\hline & Asystolie & $188(41,0)$ & $4(2,1)$ & 0,12 (vs. VF/VT) & $0,04-0,37$ & 23,38 & $<0,01$ \\
\hline & Nicht schockbar & $318(69,4)$ & $8(2,5)$ & 0,15 (vs. VF/VT) & $0,06-0,34$ & 23,10 & $<0,01$ \\
\hline \multirow{4}{*}{$\begin{array}{l}\text { Initiales } \\
\text { Atem- } \\
\text { muster } \\
\text { (n/a: } 29 \\
{[6,3 \%] \text { ) }}\end{array}$} & \multirow[t]{2}{*}{ Schnappatmung } & \multirow[t]{2}{*}{$117(25,5)$} & \multirow[t]{2}{*}{$14(12,0)$} & 3,77 (vs. Apnoe) & $1,62-8,74$ & 10,46 & 0,02 \\
\hline & & & & $\begin{array}{l}3,4 \text { (vs. keine Schnappat- } \\
\text { mung) }\end{array}$ & $1,52-7,58$ & 8,74 & $<0,01$ \\
\hline & Apnoe & $287(62,7)$ & $10(3,5)$ & $\begin{array}{l}0,27 \text { (vs. Schnappat- } \\
\text { mung) }\end{array}$ & $0,11-0,62$ & 10,46 & 0,02 \\
\hline & Beatmung & $25(5,5)$ & $2(8)$ & - & - & - & - \\
\hline \multirow{3}{*}{$\begin{array}{l}\text { Vermutete } \\
\text { Ursache }\end{array}$} & Kardial & $282(61,6)$ & $24(8,5)$ & 3,18 (vs. nicht-kardial) & $1,19-8,50$ & 6,55 & 0,01 \\
\hline & Nicht-kardial, & $176(38,4)$ & $5(2,8)$ & 0,31 (vs. kardial) & $0,12-0,84$ & 6,55 & 0,01 \\
\hline & davon klassifiziert unbekannt & $44(9,6)$ & $4(9,1)$ & - & - & - & - \\
\hline \multirow{5}{*}{$\begin{array}{l}\text { Kollaps } \\
\text { beob- } \\
\text { achtet }\end{array}$} & \multirow[t]{2}{*}{ Nicht beobachtet } & \multirow[t]{2}{*}{$174(38,0)$} & \multirow[t]{2}{*}{$4(2,3)$} & $\begin{array}{l}0,20 \text { (vs. Beob.: Nicht- } \\
\text { RettD) }\end{array}$ & $0,07-0,58$ & 13,89 & 0,01 \\
\hline & & & & 0,24 (vs. beobachtet) & $0,08-0,71$ & 8,85 & 0,01 \\
\hline & Beobachtet & 284 & $25(8,8)$ & $\begin{array}{l}4,10 \text { (vs. nicht beobach- } \\
\text { tet) }\end{array}$ & $1,40-12,00$ & 8,85 & 0,01 \\
\hline & davon: Nicht-RettD & $214(46,7)$ & $23(10,7)$ & $\begin{array}{l}5,12 \text { (vs. nicht beobach- } \\
\text { tet) }\end{array}$ & $1,74-15,10$ & 13,89 & $<0,01$ \\
\hline & davon: RettD & $70(15,3)$ & $2(2,9)$ & - & - & - & - \\
\hline \multirow{7}{*}{$\begin{array}{l}\text { Thorax- } \\
\text { kompres- } \\
\text { sion (Be- } \\
\text { ginn) }\end{array}$} & Ersthelfer & $79(17,2)$ & $12(15,2)$ & - & - & - & - \\
\hline & First Responder & $32(7,0)$ & $2(6,3)$ & - & - & - & - \\
\hline & RTW & $183(40,0)$ & $5(2,7)$ & - & - & - & - \\
\hline & Notarzt & $162(35,4)$ & $10(6,2)$ & - & - & - & - \\
\hline & Nicht durchgeführt & $2(0,4)$ & $0(0)$ & - & - & - & - \\
\hline & Nicht-RettD & $111(24,2)$ & $14(12,6)$ & 3,2 (vs. RettD) & $1,49-6,85$ & 8,47 & $<0,01$ \\
\hline & RettD & $345(75,3)$ & $15(4,3)$ & - & - & - & - \\
\hline
\end{tabular}




\begin{tabular}{|c|c|c|c|c|c|c|c|}
\hline Parameter & & $\begin{array}{l}\text { Gesamt } \\
(\%)^{\mathrm{a}}\end{array}$ & $\begin{array}{l}\mathrm{CPC} \leq 2 \\
(\%)^{\mathrm{b}}\end{array}$ & OR & $\mathrm{Cl}(95 \%)$ & $\begin{array}{l}X^{2} \text { (Mo- } \\
\text { dell) }\end{array}$ & $p\left(X^{2}\right)$ \\
\hline \multirow{6}{*}{$\begin{array}{l}\text { 1. Defi- } \\
\text { brillation } \\
\text { (n/a: } 61 \\
(13,3 \%))\end{array}$} & Ersthelfer & $2(0,4)$ & $0(0)$ & - & - & - & - \\
\hline & First Responder & $5(1,1)$ & $0(0)$ & - & - & - & - \\
\hline & RTW & $45(9,8)$ & $5(11,1)$ & 25,0 (vs. keine Defibr.) & $2,84-219,77$ & 31,96 & $<0,01$ \\
\hline & Notarzt & $144(31,4)$ & $20(13,9)$ & 32,26 (vs. keine Defibr.) & $4,28-243,37$ & 31,96 & $<0,01$ \\
\hline & Keine Defibrillation & $201(43,9)$ & $1(0,5)$ & - & - & - & - \\
\hline & Jemals Defibrillation & $196(42,8)$ & $25(12,8)$ & 29,24 (vs. keine Defibr.) & $3,92-218,05$ & 29,77 & $<0,01$ \\
\hline \multirow{6}{*}{$\begin{array}{l}\text { 1. Vaso- } \\
\text { pressor } \\
\text { (n/a: } 24 \\
(5,2 \%))\end{array}$} & Ersthelfer & $3(0,7)$ & $1(33,3)$ & - & - & - & - \\
\hline & First Responder & $6(1,3)$ & $0(0)$ & - & - & - & - \\
\hline & RTW & $4(0,9)$ & $0(0)$ & - & - & - & - \\
\hline & Notarzt & $384(83,8)$ & $18(4,7)$ & - & - & - & - \\
\hline & Kein Vasopressor & $37(8,1)$ & $5(13,5)$ & - & - & - & - \\
\hline & Jemals Vasopressor & $397(86,7)$ & $19(4,8)$ & $\begin{array}{l}0,32 \text { (vs. kein Vasopres- } \\
\text { sor) }\end{array}$ & $0,11-0,92$ & 3,73 & $<0,05$ \\
\hline \multirow{2}{*}{$\begin{array}{l}\text { Jemals } \\
\text { ROSC } \\
\text { (n/a: } 1 \\
(0,2 \%))\end{array}$} & $\mathrm{Ja}$ & $233(50,9)$ & $28(12,0)$ & 30,46 (vs. „nein“) & $4,11-225,88$ & 32,09 & $<0,01$ \\
\hline & Nein & $224(48,9)$ & $1(0,4)$ & - & - & - & - \\
\hline \multirow{6}{*}{$\begin{array}{l}\text { 1. ROSC } \\
\text { (n/a: } 204 \\
[44,5 \%])\end{array}$} & Ersthelfer & $3(0,7)$ & $2(66,7)$ & - & - & - & - \\
\hline & "First responder" & $2(0,4)$ & $0(0)$ & - & - & - & - \\
\hline & RTW & $9(2,0)$ & $2(22,2)$ & - & - & - & - \\
\hline & Notarzt & $240(52,4)$ & $24(10,0)$ & 0,06 (vs. Ersthelfer) & $0,01-0,64$ & 30,15 & $<0,01$ \\
\hline & Nicht-RettD & $5(1,1)$ & $2(40)$ & - & - & - & - \\
\hline & RettD & $249(54,4)$ & $26(10,4)$ & - & - & - & - \\
\hline \multirow{3}{*}{$\begin{array}{l}\text { Kranken- } \\
\text { hausauf- } \\
\text { nahme } \\
\text { (n/a: } 1 \\
[0,2 \%])\end{array}$} & Übergabe im ROSC & $212(46,3)$ & $27(12,7)$ & $\begin{array}{l}\text { 7,52 (vs. Übergabe unter } \\
\text { Reanimation) }\end{array}$ & $1,75-32,25$ & 34,56 & $<0,01$ \\
\hline & Übergabe unter Reanimation & $105(22,9)$ & $2(1,9)$ & - & - & - & - \\
\hline & Tod vor Aufnahme & $140(30,6)$ & - & - & - & - & - \\
\hline \multirow{6}{*}{$\begin{array}{l}\text { Bewusst- } \\
\text { seinslage } \\
\text { bei Über- } \\
\text { gabe } \\
\text { (n/a: } 32 \\
{[10,1 \%] \text { ) }}\end{array}$} & Analogsediert & $99(31,2)$ & $15(15,2)$ & 3,20 (vs. „bewusstlos") & $1,34-7,61$ & 16,51 & $<0,01$ \\
\hline & Wach (2) & $14(4,4)$ & $5(35,7)$ & 9,94 (vs. „bewusstlos“) & $2,76-35,85$ & 16,51 & $<0,01$ \\
\hline & Reaktion auf Ansprache (3) & $2(0,6)$ & $0(0)$ & - & - & - & - \\
\hline & Reaktion auf Schmerzreiz (4) & $0(0)$ & $0(0)$ & - & - & - & - \\
\hline & Bewusstlos & $170(53,6)$ & $9(5,3)$ & - & - & - & - \\
\hline & 2-4 (gesamt) & $16(5,0)$ & $5(31,3)$ & 8,13 (vs. „bewusstlos") & $2,32-28,45$ & 12,99 & $<0,01$ \\
\hline \multicolumn{8}{|c|}{$\begin{array}{l}\text { OR, CI Odds Ratio und Konfidenzintervall ( } 95 \% \text { ) in der univariaten Analyse (versus Komparator) (s. Text) } \\
\text { Beob. beobachtet, CPC Cerebral Performance Category, Defibr. Defibrillation, } n / a \text { keine oder nicht verwertbare Angabe, RettD Rettungsdienst, ROSC "re- } \\
\text { turn of spontaneous circulation“, RTW Rettungswagen, VFNT ventrikuläres Flimmern, ventrikuläre Tachykardie, vs. versus } \\
\text { anteil (\%) am Gesamtkollektiv } \\
{ }^{b} \mathrm{CPC} \leq 2 \text {-Ergebnis, Anteil von }{ }^{\mathrm{a}}\end{array}$} \\
\hline
\end{tabular}

\section{Allgemeiner Gesundheitszustand - Fragebogen}

Der versendete Fragebogen war inhaltlich an den SF-12-Fragebogen angelehnt, wurde allerdings adaptiert (Suppl.-Tab. 1).

Insgesamt stuften 23 Personen ihren Gesundheitszustand als positiv ("gut" bis „ausgezeichnet ${ }^{\prime \prime}$ ) ein, das entspricht 74,2\% aller 31 gültigen Antworten. Es ergeben sich drei weitere Szenarien: 1. Wird ein Überleben nicht nachverfolgbarer Patienten bei schlechter Gesundheit angenom- men, so ergibt sich ein positiver Gesundheitszustand in insgesamt 43,4\% (23/53). 2. Wird ein Überleben dieser Patienten bei guter Gesundheit angenommen, so ergibt sich ein positiver Gesundheitszustand in insgesamt 84,9\% (45/53). 3. Wird davon ausgegangen, dass diese Patienten verstarben, so erreichten insgesamt $67,6 \%$ der Überlebenden einen „guten“ Gesundheitszustand (23/34).

In der weiteren Selbsteinschätzung der Patienten wurden allgemein-körperliche, neurologische und seelisch-emotionale
Gesundheit überwiegend gut bewertet, es bestanden keine bis mäßige Funktionseinschränkungen (Suppl.-Tab. 1). Auf 18 der 33 (54,5\%) zurückgesendeten Fragebögen wurde die Frage, ob sie anderen Menschen empfehlen würden, einen Reanimationsversuch nach OHCA zuzulassen, beantwortet. Alle 18 Patienten bejahten diese Frage. 
Tab. 2 Analysierte Parameter im Kollektiv der Patienten, die mit ROSCaufgenommen wurden

\begin{tabular}{|c|c|c|c|c|c|c|c|}
\hline Parameter & & $\begin{array}{l}\text { Gesamt } \\
(\%)^{\mathrm{a}}\end{array}$ & $\begin{array}{l}\mathrm{CPC} \leq 2 \\
(\%)^{\mathrm{b}}\end{array}$ & OR & $\mathrm{Cl}(95 \%)$ & $\begin{array}{l}X^{2} \text { (Mo- } \\
\text { dell) }\end{array}$ & $p\left(X^{2}\right)$ \\
\hline $\begin{array}{l}\text { Alter (in } \\
\text { Jahren) }\end{array}$ & - & $\varnothing 69,1$ & $\varnothing 58,7$ & 0,95 & $0,92-0,98$ & 14,55 & $<0,01$ \\
\hline \multirow[t]{2}{*}{ Geschlecht } & Weiblich & $76(35,7)$ & $7(9,2)$ & - & - & - & - \\
\hline & Männlich & $137(64,3)$ & $20(14,6)$ & - & - & - & \\
\hline \multirow{12}{*}{$\begin{array}{l}\text { Einsatzort } \\
\text { (n/a: } 1 \\
[0,5 \%])\end{array}$} & Wohnung (1) & $118(55,7)$ & $10(8,5)$ & 0,30 (vs. öffentlich komb.) & $0,12-0,75$ & 17,69 & $<0,01$ \\
\hline & Altenpflegeeinrichtung (2) & $25(11,8)$ & $0(0)$ & - & - & - & - \\
\hline & Arbeitsplatz (3) & $7(3,3)$ & $3(42,9)$ & - & - & - & - \\
\hline & Arztpraxis (4) & $4(1,9)$ & $2(50,0)$ & 10,80 (vs. Wohnung) & $1,37-85,10$ & 17,69 & $<0,01$ \\
\hline & Straße (5) & $24(11,3)$ & $5(20,8)$ & - & - & - & - \\
\hline & Öffentlicher Raum (6) & $15(7,1)$ & $2(13,3)$ & - & - & - & - \\
\hline & Krankenhaus (7) & $9(4,2)$ & $2(22,2)$ & - & - & - & - \\
\hline & Massenveranst. (8) & $1(0,5)$ & $0(0)$ & - & - & - & - \\
\hline & Sonstiges (9) & $4(1,9)$ & $1(25,0)$ & - & - & - & - \\
\hline & Sportstätte (11) & $4(1,9)$ & $2(50)$ & - & - & - & - \\
\hline & Öffentl. kombiniert I $(3,5,6,8,11)$ & $51(24,1)$ & $12(23,5)$ & 3,32 (vs. Wohnung) & $1,33-8,30$ & 17,69 & $<0,01$ \\
\hline & Öffentl. kombiniert II $(3,4,5,6,8,11)$ & $55(25,9)$ & $14(25,5)$ & 3,76 (vs. Rest) & $1,64-8,62$ & 9,52 & $<0,01$ \\
\hline \multirow{5}{*}{$\begin{array}{l}\text { Initialer } \\
\text { Rhythmus }\end{array}$} & \multirow[t]{2}{*}{ VF/VT } & \multirow[t]{2}{*}{$88(41,5)$} & \multirow[t]{2}{*}{$19(21,6)$} & 4,82 (vs. Asystolie) & $1,56-14,89$ & 10,87 & $<0,01$ \\
\hline & & & & 3,99 (vs. nicht schockbar) & $1,66-9,61$ & 10,54 & $<0,01$ \\
\hline & PEA & $50(23,6)$ & $4(8,0)$ & 0,32 (vs. VF/VT) & $0,10-0,99$ & 10,87 & $<0,01$ \\
\hline & Asystolie & $74(34,9)$ & $4(5,4)$ & 0,21 (vs. VF/VT) & $0,07-0,64$ & 10,87 & $<0,01$ \\
\hline & Nicht schockbar & $124(58,5)$ & $8(6,5)$ & 0,28 (vs. VF/VT) & $0,10-0,60$ & 10,54 & $<0,01$ \\
\hline \multirow{3}{*}{$\begin{array}{l}\text { Initiales } \\
\text { Atemmuster } \\
\text { (n/a: } 17 \\
[8,0 \%])\end{array}$} & Schnappatmung & $67(31,6)$ & $12(17,9)$ & - & - & - & - \\
\hline & Apnoe & $119(56,1)$ & $10(8,4)$ & - & - & - & - \\
\hline & Beatmung & $9(4,2)$ & $2(22,2)$ & - & - & - & - \\
\hline \multirow{3}{*}{$\begin{array}{l}\text { Vermutete } \\
\text { Ursache }\end{array}$} & Kardial & $133(62,7)$ & $23(17,3)$ & 3,92 (vs. nichtkardial) & $1,30-11,80$ & 7,53 & $<0,01$ \\
\hline & Nichtkardial & $79(37,3)$ & $4(5,1)$ & 0,26 (vs. kardial) & $0,09-0,77$ & 7,53 & $<0,01$ \\
\hline & Davon klassifiziert unbekannt & $19(9,0)$ & $3(15,8)$ & - & - & - & - \\
\hline \multirow[t]{4}{*}{$\begin{array}{l}\text { Kollaps } \\
\text { beobachtet }\end{array}$} & Nicht beobachtet & $64(30,2)$ & $4(6,3)$ & $\begin{array}{l}0,31 \text { (vs. beob.: } \\
\text { Nicht-RettD) 0,36 (vs. } \\
\text { beobachtet) }\end{array}$ & $\begin{array}{l}0,10-0,9 \\
0,12-1,09\end{array}$ & $6,553,90$ & $\begin{array}{l}0,04 \\
<0,05\end{array}$ \\
\hline & Beobachtet & $148(69,8)$ & $23(15,5)$ & 2,76 (vs. nicht beobachtet) & $0,91-8,34$ & 3,90 & $<0,05$ \\
\hline & - davon: Nicht-RettD & $118(55,7)$ & $21(17,8)$ & 2,76 (vs. nicht beobachtet) & $1,06-9,92$ & 6,55 & 0,04 \\
\hline & - davon: RettD & $30(14,2)$ & $2(6,7)$ & - & & & \\
\hline \multirow{7}{*}{$\begin{array}{l}\text { Thorax- } \\
\text { kompres- } \\
\text { sion (Beginn) }\end{array}$} & Ersthelfer & $45(21,1)$ & $10(22,2)$ & - & - & - & - \\
\hline & „First responder" & $13(6,1)$ & $2(15,4)$ & - & - & - & - \\
\hline & RTW & $84(39,6)$ & $5(6,0)$ & - & - & - & - \\
\hline & Notarzt & $70(32,5)$ & $10(14,5)$ & - & - & - & - \\
\hline & Nicht durchgeführt & $1(0,5)$ & $0(0)$ & - & - & - & - \\
\hline & Nicht-RettD & $58(27,3)$ & $12(20,7)$ & 2,42 (vs. Rest) & $1,06-5,54$ & 4,19 & 0,04 \\
\hline & RettD & $153(72,4)$ & $15(9,8)$ & - & - & - & - \\
\hline \multirow{6}{*}{$\begin{array}{l}\text { 1. Defibrilla- } \\
\text { tion } \\
\text { (n/a: } 32 \\
[15,1 \%])\end{array}$} & Ersthelfer & $1(0,5)$ & $0(0)$ & - & - & - & - \\
\hline & „First responder" & $3(1,4)$ & $0(0)$ & - & - & - & - \\
\hline & RTW & $28(13,2)$ & $5(17,9)$ & 14,13 (vs. keine Defibr.) & $1,57-127,41$ & 18,41 & $<0,01$ \\
\hline & Notarzt & $82(38,7)$ & $18(22,0)$ & 18,28 (vs. keine Defibr.) & $2,37-141,02$ & 18,41 & $<0,01$ \\
\hline & Keine Defibrillation & $66(31,1)$ & $1(1,5)$ & - & - & - & - \\
\hline & Jemals Defibrillation & $114(53,5)$ & $23(20,2)$ & 16,43 (vs. keine Defibr.) & $2,16-124,75$ & 16,36 & $<0,01$ \\
\hline
\end{tabular}




\begin{tabular}{|c|c|c|c|c|c|c|c|}
\hline Parameter & & $\begin{array}{l}\text { Gesamt } \\
(\%)^{\mathrm{a}}\end{array}$ & $\begin{array}{l}\mathrm{CPC} \leq 2 \\
(\%)^{\mathrm{b}}\end{array}$ & OR & $\mathrm{Cl}(95 \%)$ & $\begin{array}{l}\mathrm{X}^{2} \text { (Mo- } \\
\text { dell) }\end{array}$ & $p\left(X^{2}\right)$ \\
\hline \multirow{6}{*}{$\begin{array}{l}\text { 1. Vasopres- } \\
\text { sor } \\
\text { (n/a: } 13 \\
[6,1 \%])\end{array}$} & Ersthelfer & $3(1,4)$ & $1(33,3)$ & - & - & - & - \\
\hline & „First responder" & $3(1,4)$ & $0(0)$ & - & - & - & - \\
\hline & RTW & $2(0,9)$ & $0(0)$ & - & - & - & - \\
\hline & Notarzt & $173(81,1)$ & $16(9,2)$ & - & - & - & - \\
\hline & Kein Vasopressor & $19(9,0)$ & $5(26,3)$ & - & - & - & - \\
\hline & Jemals Vasopressor & $181(85,0)$ & $17(9,4)$ & 0,29 (vs. kein Vasopressor) & $0,09-0,91$ & 3,90 & $<0,05$ \\
\hline \multirow{6}{*}{$\begin{array}{l}\text { 1. ROSC } \\
\text { (n/a: } 2 \\
[0,9 \%])\end{array}$} & Ersthelfer & $3(1,4)$ & $2(66,7)$ & - & - & - & - \\
\hline & „First responder" & $1(0,5)$ & $0(0)$ & - & - & - & - \\
\hline & RTW & $8(3,8)$ & $2(25,0)$ & - & - & - & - \\
\hline & Notarzt & $198(93,4)$ & $23(11,6)$ & - & - & - & - \\
\hline & Nicht-RettD & $4(1,9)$ & $2(50,0)$ & - & - & - & - \\
\hline & RettD & $206(97,2)$ & $25(12,1)$ & - & - & - & - \\
\hline \multirow{6}{*}{$\begin{array}{l}\text { Bewusst- } \\
\text { seins- } \\
\text { lage bei } \\
\text { Überga- } \\
\text { be (n/a: } 11 \\
[5,2 \%])\end{array}$} & Analgosediert & $96(45,3)$ & $15(15,6)$ & - & - & - & - \\
\hline & Wach (2) & $14(6,6)$ & $5(35,7)$ & - & - & - & - \\
\hline & Reaktion auf Ansprache (3) & $2(0,9)$ & $0(0)$ & - & - & - & - \\
\hline & Reaktion auf Schmerzreiz (4) & $0(0)$ & - & - & - & - & - \\
\hline & Bewusstlos & $89(42,0)$ & $7(7,9)$ & - & - & - & - \\
\hline & "Wach" (2-4) & $16(7,5)$ & $5(31,3)$ & 5,33 (vs. „bewusstlos“) & $1,44-19,71$ & 6,48 & 0,04 \\
\hline
\end{tabular}

\section{Diskussion}

In der vorliegenden Arbeit beschreiben wir das Überleben und neurologische Langzeitergebnis aus einem Kollektiv von 458 reanimierten Patienten $\geq 2,5$ Jahre nach OHCA. Des Weiteren beschreiben wir den von den Patienten selbst eingeschätzten Gesundheitszustand bei Langzeitüberleben. Nach unserer Kenntnis ist dies die erste Arbeit, die das neurologische Langzeitergebnis nach OHCA in Deutschland in dieser Form darstellt und mögliche Zusammenhänge zu den standardisiert erhobenen Parametern statistisch untersucht.

Die Reanimationsinzidenz nach OHCA im Rettungsdienst der Stadt Bonn lag mit 57/100.000/Jahr unter der für Deutschland in der EuReCa-ONE-Studie veröffentlichten Inzidenz von 66/100.000/Jahr, die einen ähnlichen Beobachtungszeitraum umfasste [10]. Im Deutschen Reanimationsregister wird eine ROSC-Rate von $44,8 \%$, eine Krankenhausaufnahme mit ROSC in 37,6\% und ein Überleben bis Entlassung (oder bis 30 Tage) in ca. 13,1\% der Fälle (davon $68,3 \%$ mit CPC $\leq 2$ ) angegeben (Untersuchungszeitraum 2007-2017; [9]). Eine an- dere Studie beschreibt eine Krankenhausentlassungsrate von $13 \%$ (Untersuchungszeitraum 2010-2016; [5]). Die in der vorliegenden Arbeit beschriebenen Überlebensraten im städtisch geprägten Bonner Rettungsdienst interpretieren wir als insgesamt plausibel (Überleben bis Krankenhausentlassung 17,9\%).

Studien zum neurologischen Langzeitergebnis nach Krankenhausentlassung nach OHCA sind für Deutschland kaum verfügbar. Daten aus anderen Rettungsdienstsystemen zeigen, dass ein gutes neurologisches Ergebnis ein Jahr nach versuchter Reanimation nach OHCA in 2,8\% (Japan; [28]), 5,4\% (Australien; [26]) und 6,6-7,3\% (Dänemark; [16, 27]) erreicht wurde. Es ist zu berücksichtigen, dass Sondergaard et al. nur Fälle mit vermuteter nichtkardialer Ursache des OHCA und Fälle mit vorbekannter neurologischer Beeinträchtigung aus ihrer Analyse ausschlossen [27]. Auch Kragholm et al. schlossen insgesamt 18,1\% aller Patienten aus ihrer Analyse aus [16]. Hingegen wurde in unserer Betrachtung das Fehlen von Daten als nicht zufällig beurteilt (statistisch: „missing not at ran- dom"). Diese Fälle wurden nicht aus der Untersuchung ausgeschlossen, sondern als anzunehmend "schlechtes" Ergebnis ( $C P C \geq 3$ bzw. verstorben) berücksichtigt.

Darüber hinaus wurde das neurologische Langzeitergebnis in unserer Arbeit durch Befragung der Patienten direkt ermittelt. Auch die Zufriedenheit der Patienten mit dem Reanimationsergebnis insgesamt wurde abgefragt: Alle Patienten, die die entsprechende Frage beantworteten, würden anderen Menschen empfehlen, einen Reanimationsversuch nach OHCA zuzulassen. Dementsprechend wurde auch der eigene Gesundheitszustand von einem großen Teil der überlebenden Patienten positiv bewertet. Hierbei wurden der körperliche, geistig-kognitive und auch emotionale Gesundheitszustand ähnlich beurteilt.

Es ist versucht worden, das Überleben nach OHCA und auch das neurologische Ergebnis zu prognostizieren: Sasson et al. beschrieben, dass ein Überleben von der Beobachtung des Kollapses, der Durchführung von Basismaßnahmen durch Ersthelfer, dem initialen Rhythmus und dem präklinischen Wiedererlangen ei- 
Tab. 3 Analysierte Parameter im Gesamtkollektiv aller reanimierten Patienten

\begin{tabular}{|c|c|c|c|c|}
\hline \multirow{3}{*}{$\begin{array}{l}\text { Multivariate Analyse } \\
\text { Gesamt }\end{array}$} & \multicolumn{2}{|c|}{ 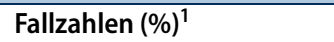 } & \multirow{2}{*}{$\begin{array}{l}X^{2} \text { (Modell) } \\
49,48\end{array}$} & \multirow{2}{*}{$\begin{array}{l}\mathrm{p}\left(\mathrm{X}^{2}\right) \\
<0,01\end{array}$} \\
\hline & Einbezogen: & $367(80,1)^{a}$ & & \\
\hline & Fehlend: & $91(19,9)^{a}$ & $43,85^{b}$ & $<0,01^{b}$ \\
\hline Parameter & & & OR & $\mathrm{Cl}(95 \%) / p$ \\
\hline Alter (in Jahren) & & & 0,96 & $0,931-0,004 / 0,019$ \\
\hline \multirow[t]{2}{*}{$\begin{array}{l}\text { Schnappatmung (bei } \\
\text { Eintreffen) }\end{array}$} & Ja: & $98(26,7)$ & $\begin{array}{l}2,51 \\
2,81^{b}\end{array}$ & $\begin{array}{l}0,92-6,83 / 0,07 \\
1,06-7,42 / 0,04^{b}\end{array}$ \\
\hline & Nein: & $269(73,3)$ & - & - \\
\hline \multirow[t]{2}{*}{ Kollaps beobachtet } & Ja: & $136(37,1)$ & $\begin{array}{l}12,63 \\
9,84^{b}\end{array}$ & $\begin{array}{l}1,54-103,71 / 0,02 \\
1,26-76,92 / 0,03^{b}\end{array}$ \\
\hline & Nein: & $231(62,9)$ & - & - \\
\hline \multirow[t]{2}{*}{ Defibrillation } & Jemals: & $175(47,7)$ & $\begin{array}{l}14,22 \\
19,43^{b}\end{array}$ & $\begin{array}{l}1,82-110,90 / 0,01 \\
2,52-149,73 /<0,01^{b}\end{array}$ \\
\hline & Niemals: & $192(52,3)$ & - & - \\
\hline \multirow[t]{2}{*}{ Vasopressor } & Jemals: & $334(91,0)$ & $\begin{array}{l}0,23 \\
0,24^{b}\end{array}$ & $\begin{array}{l}0,06-0,85 / 0,03 \\
0,07-0,85 / 0,03^{b}\end{array}$ \\
\hline & Niemals: & $33(9,0)$ & - & - \\
\hline ROC-Kurve & Sensitivität & Spezifität & \multicolumn{2}{|c|}{ AUC (Cl [95\%]) } \\
\hline \multirow[t]{2}{*}{-} & 0,91 & 0,78 & \multicolumn{2}{|c|}{$0,90(0,84-0,95)$} \\
\hline & $0,91^{\mathrm{b}}$ & $0,70^{b}$ & \multicolumn{2}{|c|}{$0,87(0,80-0,95)^{b}$} \\
\hline \multicolumn{5}{|c|}{ 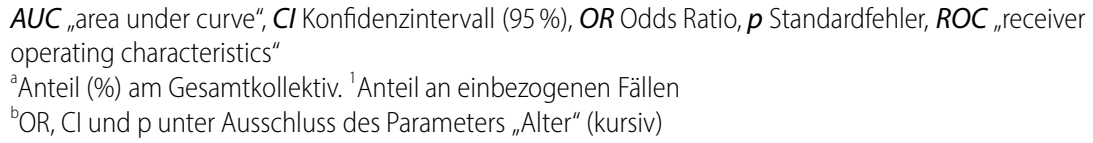 } \\
\hline
\end{tabular}

Tab. 4 Analysierte Parameter im Kollektiv der Patienten, die mit ROSCaufgenommen wurden

\begin{tabular}{|c|c|c|c|c|}
\hline Multivariate Analyse & \multicolumn{2}{|c|}{ Fallzahlen $(\%)^{1}$} & $\mathrm{X}^{2}$ (Modell) & $p\left(X^{2}\right)$ \\
\hline \multirow[t]{2}{*}{ Gesamt } & Einbezogen: & $160(75,5)^{\mathrm{a}}$ & 33,03 & $<0,01$ \\
\hline & Fehlend: & $52(24,5)^{\mathrm{a}}$ & $26,60^{b}$ & $<0.01^{b}$ \\
\hline \multicolumn{3}{|l|}{ Parameter } & OR & $\mathrm{Cl}(95 \%) / p$ \\
\hline \multicolumn{3}{|l|}{ Alter (in Jahren) } & 0,95 & $0,91-0,99 / 0,01$ \\
\hline \multirow[t]{2}{*}{$\begin{array}{l}\text { Schnappatmung (bei } \\
\text { Eintreffen) }\end{array}$} & Ja: & $57(35,6)$ & $\begin{array}{l}1,68 \\
1,79^{b}\end{array}$ & $\begin{array}{l}0,55-5,15 / 0,37 \\
0,61-5,22 / 0,29^{b}\end{array}$ \\
\hline & Nein: & $103(64,4)$ & - & - \\
\hline \multirow[t]{2}{*}{ Kollaps beobachtet } & Ja: & $116(72,5)$ & $\begin{array}{l}11,54 \\
8,28^{b}\end{array}$ & $\begin{array}{l}1,36-98,21 / 0,03 \\
1,03-66,34 /<0,05^{b}\end{array}$ \\
\hline & Nein: & $44(27,5)$ & - & - \\
\hline \multirow[t]{2}{*}{ Defibrillation } & Jemals: & $97(60,6)$ & $\begin{array}{l}9,20 \\
14,86^{b}\end{array}$ & $\begin{array}{l}1,13-74,87 / 0,04 \\
1,88-117,77 / 0,01^{b}\end{array}$ \\
\hline & Niemals: & $63(39,4)$ & - & - \\
\hline \multirow[t]{2}{*}{ Vasopressor } & Jemals: & $145(90,6)$ & $\begin{array}{l}0,29 \\
0,29^{b}\end{array}$ & $\begin{array}{l}0,06-1,28 / 0,10 \\
0,07-1,13 / 0,07^{b}\end{array}$ \\
\hline & Niemals: & $15(9,4)$ & - & - \\
\hline ROC-Kurve & Sensitivität & Spezifität & \multicolumn{2}{|c|}{ AUC (CI [95\%]) } \\
\hline \multirow[t]{2}{*}{-} & 0,90 & 0,74 & \multicolumn{2}{|c|}{$0,87(0,81-0,94)$} \\
\hline & $0,90^{b}$ & $0,64^{b}$ & \multicolumn{2}{|c|}{$0,84(0,74-0,93)^{b}$} \\
\hline
\end{tabular}

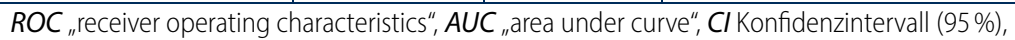
OR Odds Ratio, $p$ Standardfehler

${ }^{a}$ Anteil (\%) am Gesamtkollektiv. ${ }^{1}$ Anteil an einbezogenen Fällen

${ }^{\mathrm{O}} \mathrm{OR}, \mathrm{Cl}$ und $\mathrm{p}$ unter Ausschluss des Parameters „Alter" (kursiv) nes Spontankreislaufs abhängt [24]. Auf Grundlage des Deutschen Reanimationsregisters erarbeiteten Seewald et al. den CaRdiac Arrest Survival Score (CRASS; [25]): Im Kollektiv aller aufgenommenen Patienten ist eine Entlassung mit einem guten neurologischen Ergebnis wahrscheinlicher mit jüngerem Patientenalter, beobachtetem Kollaps, Vorliegen von VF/VT, schnellem Beginn einer CPR, fehlenden oder nur geringfügigen Vorerkrankungen, Arrest an bestimmten Orten (Öffentlichkeit, Arztpraxis), der Gabe von Amiodaron, kurzer CPRDauer und Normotension bei Krankenhausaufnahme. Die Wahrscheinlichkeit eines guten neurologischen Überlebens bis zur Entlassung wird demnach verringert durch höheres Alter, PEA oder Asystolie, ursächliches Trauma, Notwendigkeit einer mechanischen Reanimation, Adrenalingabe, Pflegebedürftigkeit, Aufnahme unter CPR und längerer CPR.

Übereinstimmend mit den oben zitierten Studien war in der multivariaten logistischen Regressionsanalyse unserer Daten ein gutes neurologisches Langzeitergebnis abhängig von einem geringeren Alter, einem beobachteten Kollaps, der Durchführung einer Defibrillation während der Reanimation, dem Vorliegen von Schnappatmung, sowie dem Ausbleiben einer Vasopressorgabe. Aus der Assoziation der Gabe eines Vasopressors mit einer niedrigeren Wahrscheinlichkeit für ein gutes neurologisches Langzeitergebnis kann kein kausaler Zusammenhang abgeleitet werden. Vielmehr interpretieren wir die Gabe von Adrenalin als indirektes Anzeichen einer länger dauernden Reanimation oder aber eines primär nicht schockbaren Rhythmus. In beiden Fällen ist ein gutes neurologisches Ergebnis unwahrscheinlich [4, 25], was mit den Ergebnissen der PARAMEDIC2Studie übereinstimmt [23]. Dass kein Zusammenhang anderer Parameter mit dem Outcome gezeigt werden konnte, lässt sich hinreichend mit der Abhängigkeit einzelner Variablen voneinander erklären. So ist z.B. ein Zusammenhang zwischen dem initialen Rhythmus, der Durchführung einer Bystander-CPR und der Frage, ob der Kollaps beobachtet wurde, anzunehmen. Darüber hinaus wurden die Parameter in unserer Analyse in dichotome Variablen transformiert, beispielsweise wurde der Parameter "1. Defibrillation“ (durch Erst- 
helfer, RTW, NEF etc.) in „Defibrillation ja/nein" überführt.

Da das Langzeitüberleben offensichtlich durch die Lebenserwartung des Patienten vor OHCA beeinflusst wird, analysierten wir die Kollektive auch unter Ausschluss des Parameters „Alter", was das übrige Ergebnis allerdings nicht beeinflusste.

In der univariaten logistischen Regressionsanalyse unserer Studie war ein gutes neurologisches Ergebnis zusätzlich wahrscheinlicher bei Aufenthalt an öffentlichen oder anderen ausgewählten Orten, dem initialen Vorliegen von VF/VT, dem Beginn von Thoraxkompressionen durch Ersthelfer, dem Erreichen von ROSC, ROSC bei Aufnahme und dem Wiedererlangen des Bewusstseins bis zur Aufnahme. Kein Patient erreichte nach Reanimation in einer Altenpflegeeinrichtung ein gutes Langzeitergebnis. Deshalbscheint die vorausschauende, patientenindividuelle Erstellung von Handlungsanweisungen in dieser Population besonders geboten [12].

In unserer Studie erzielten nur 1,9\% der unter Reanimation ins Krankenhaus aufgenommenen Patienten ein gutes neurologisches Ergebnis. Unsere Ergebnisse stehen nicht im Widerspruch zu einer aktuellen Kölner Studie, in der unter CPR transportierte Patienten ein besseres neurologisches Outcome zeigten [1]. Während in unserer Studie die Ergebnisse der notärztlichen Realversorgung retrospektiv berichtet sind, wurden dort selektiert nur solche Patienten mit einem refraktären OHCA transportiert, die 18-75 Jahre alt waren, deren Kollaps beobachtet worden war, die initial einen schockbaren Rhythmus aufwiesen oder im Falle von PEA/Asystolie im Beisein des Rettungsdiensts kollabierten, die eine No-flow-Zeit von weniger als fünf Minuten hatten, deren Arrest-Ursache mutmaßlich kardial bedingt war und die eine mutmaßlich effektive Bystander-CPR erhalten hatten. In einer weiteren Studie desselben Rettungsdiensts (Köln) konnte gezeigt werden, dass $50 \%$ aller unter CPR transportierten Patienten überlebten (Krankenhausentlassung; [4]). Hierbei handelte es sich wiederum um ein selektioniertes Patientenkollektiv (primär refraktärer schockbarer Rhythmus), Patienten mit nicht schockbaren Rhythmen wurden ausgeschlossen.

\section{Limitationen}

Die Qualität der hier analysierten Registerdaten weist Limitationen auf: Jeder einzelne Notarzt war verantwortlich für die von inm eingegebenen Parameter. Eine nachträgliche Korrektur fehlerhafter oder nicht eingetragener Daten erfolgte nicht. Die korrekte Erhebung einzelner Parameter kann angezweifelt werden. So sind Unschärfen z.B. hinsichtlich des Atemmusters, des Vorliegens einer Bewusstlosigkeit bei Übergabe oder auch hinsichtlich des Vorliegens eines ROSC denkbar. Unvollständige Datensätze erschweren eine korrekte Auswertung und Interpretation, z. B. ob ein Vasopressor während eines Reanimationsversuchs oder erst in der Postreanimationsphase gegeben wurde. Weitere, u.U. für das Ergebnis relevante Parameter werden durch das Reanimationsregister gar nicht erhoben (z.B. Qualität der Thoraxkompressionen).

Eine weitere Limitation unserer Studie war der Anteil nicht nachzuverfolgender Patienten. Wir postulieren, dass die entsprechend fehlenden Daten nicht zufällig fehlen (statistisch: "missing not at random"), sondern dass ein kausaler Zusammenhang zwischen fehlenden Überlebens- bzw. neurologischen Outcome-Daten und dem entsprechenden Datenwert existiert. Es besteht eine hohe Wahrscheinlichkeit für ein "selection bias" [14], ein „Teilnahmebias" [8] und möglicherweise auch das Vorliegen eines „disability paradox" [3]. Es ist anzunehmen, dass ein Teil der Fragebögen nicht beantwortet wurde, weil ein schlechtes Outcome vorlag bzw. Patienten mit schlechtem CPC sich einerEinschätzung verwehrten. Aus diesen Gründen wurden die fehlenden Ergebnisse methodologisch zur weiteren statistischen Analyse als CPC $\geq 3$ eingestuft. Dies stellt jedoch lediglich eine Annahme dar.

Der durch die Patienten selbst beurteilte Gesundheitszustand ist hochgradig subjektiv und unterliegt einem Adaptationsprozess, der auch bei schwerwiegender Behinderung eine gute subjektive Lebensqualität ermöglicht. Es muss also zwischen "subjektiver" und "funktioneller" Einschränkung unterschieden werden. Hierüber ermöglichte die uns vorliegende Datengrundlage aber keine Einschätzung. Darüber hinaus sind in dieser Studie, wie auch in zahlreichen anderen Reanimationsstudien, keine Angaben zu etwaigen Komorbiditäten der Patienten erfasst, die ebenso das Behandlungsergebnis beeinflusst haben könnten. Ebenso wurden in der Studie keine Aspekte zu etwaigen Patientenwünschen (z.B. Einschränkungen der Intensivtherapie laut Patientenverfügung) erfasst, die im Behandlungsverlauf das weitere Outcome einer Reanimation beeinflusst haben könnten und somit auch insgesamt die Ergebnisse dieser Studie.

Aufgrund des hier gewählten monozentrischen Studiendesigns bleibt unklar, inwieweit die vorgelegten Studienergebnisse Rückschlüsse für andere Versorgungsbereiche zulassen.

\section{Fazit für die Praxis}

- Langzeitüberlebende eines OHCA in einem städtischen Versorgungsbereich zeigen ein gutes neurologisches Langzeitergebnis. Dieses Ergebnis scheint von denselben klinischen Parametern wie der primäre Reanimationserfolg abzuhängen.

- Dabei bewertet ein großer Teil der befragten Langzeitüberlebenden den eigenen Gesundheitszustand positiv.

- Prognoseinstrumente könnten zur frühzeitigen Abschätzung eines funktionellen Outcomes nützlich sein, auch als Hilfestellung zur Wahrung der Patientenautonomie im Verlauf einer (Post-)Reanimationsbehandlung (z. B. CRASS).

- Zusammenfassend sehen wir unsere Ergebnisse als Motivation zur Initiation größerer Studien, um die Indikation zu Durchführung und Abbruch eines Reanimationsversuchs situations- und patientengerecht beurteilen zu können.

\section{Korrespondenzadresse}

\section{Dr. med. J.-C. Schewe}

Klinik für Anästhesiologie und Operative Intensivmedizin, Universitätsklinikum Bonn, Venusberg-Campus 1 53127 Bonn, Deutschland

jens-christian.schewe@ukbonn.de

Funding. Open Access funding enabled and organized by Projekt DEAL.

\section{Einhaltung ethischer Richtlinien}

Interessenkonflikt. J.M. Poth, C.M. Buschmann, J. Kappler, U. Heister, S.F. Ehrentraut, S. Muenster, C.J. Diepenseifen, R. Ellerkmann und J.-C. Schewe geben an, dass kein Interessenkonflikt besteht. 
Das vorliegende Studiendesign wurde durch die Ethikkommission der Medizinischen Fakultät der Rheinischen Friedrich-Wilhelms-Universität Bonn genehmigt (Lfd. Nr. 034/17).

Open Access. Dieser Artikel wird unter der Creative Commons Namensnennung 4.0 International Lizenz veröffentlicht, welche die Nutzung, Vervielfältigung, Bearbeitung, Verbreitung und Wiedergabe in jeglichem Medium und Format erlaubt, sofern Sie den/die ursprünglichen Autor(en) und die Quelle ordnungsgemäß nennen, einen Link zur Creative Commons Lizenz beifügen und angeben, ob Änderungen vorgenommen wurden.

Die in diesem Artikel enthaltenen Bilder und sonstiges Drittmaterial unterliegen ebenfalls der genannten Creative Commons Lizenz, sofern sich aus der Abbildungslegende nichts anderes ergibt. Sofern das betreffende Material nicht unter der genannten Creative Commons Lizenz steht und die betreffende Handlung nicht nach gesetzlichen Vorschriften erlaubt ist, ist für die oben aufgeführten Weiterverwendungen des Materials die Einwilligung des jeweiligen Rechteinhabers einzuholen.

Weitere Details zur Lizenz entnehmen Sie bitte der Lizenzinformation auf http://creativecommons.org/ licenses/by/4.0/deed.de.

\section{Literatur}

1. Adler C, Paul C, Michels G et al (2019) One year experience with fast track algorithm in patients with refractory out-of-hospital cardiac arrest. Resuscitation 144:157-165. https://doi.org/10. 1016/j.resuscitation.2019.07.035

2. BeesemsSG, Wittebrood KM, deHaan RJ, KosterRW (2014) Cognitive function and quality of life after successful resuscitation from cardiac arrest. Resuscitation 85:1269-1274. https://doi.org/10. 1016/j.resuscitation.2014.05.027

3. Blome C, Augustin M (2015) Measuring change in quality of life: bias in prospective and retrospective evaluation. Value Health 18:110-115. https://doi. org/10.1016/j.jval.2014.10.007

4. Braumann S, Nettersheim FS, Hohmann C et al (2020) How long is long enough? Good neurologic outcome in out-of-hospital cardiac arrest survivors despite prolonged resuscitation: a retrospective cohort study. Clin Res Cardiol. https://doi.org/10. 1007/s00392-020-01640-x

5. Bürger A, Wnent J, Bohn A et al (2018) The effect of ambulance response time on survival following out-of-hospital cardiac arrest. Dtsch Aerzteblatt Online. https://doi.org/10.3238/arztebl.2018. 0541

6. Deakin CD, Nolan JP, Soar J et al (2010) Erweiterte Reanimationsmaßnahmen für Erwachsene („,advanced life support ${ }^{\prime \prime}$ ): Sektion 4 der Leitlinien zur Reanimation 2010 des European Resuscitation Council. Notfall Rettungsmed 13:559-620. https:// doi.org/10.1007/s10049-010-1370-3

7. van Diepen S, Girotra S, Abella BS et al (2017) Multistate 5-year initiative to improve care for outof-hospital cardiac arrest: primary results from the heartrescue project. J Am Heart Assoc. https://doi. org/10.1161/JAHA.117.005716

8. Fowler F (2009) Survey research methods, 4. Aufl. https://doi.org/10.4135/9781452230184

9. Gässler H, Fischer M, Wnent J et al (2019) Outcome after pre-hospital cardiac arrest in accordance with underlying cause. Resuscitation 138:36-41.

\section{Neurological outcome and general health status of long-term survivors after out-of-hospital cardiac arrest}

Objective: For Germany, few data are available on health status and long-term survival after out-of-hospital cardiac arrest. The present study investigates the general health status and long-term neurological outcome 2.5-5 years after the resuscitation attempt, as well as the association between treatment outcome and prehospital patient and treatment factors.

Methods: All resuscitation attempts performed over a period of 30 months (2011-2013) by the emergency medical services of the city of Bonn were retrospectively analyzed on the basis of treatment records and the German Resuscitation Registry. General health status was recorded following the SF-12 (12-item Short Form Health Survey) and longterm neurological outcome was categorized (Cerebral Performance Category [CPC]). The available data were subjected to univariate and multivariate logistic regression analysis.

Results: Of a total of 458 patients, $17.9 \%$ survived to hospital discharge, $13.8 \%$ survived more than 2.5 years, and $7.7 \%$ survived to the cutoff date of the survey. Of the surviving patients, $85.3 \%$ had a good neurological outcome (CPC $\leq 2)$, which was favored by lower age, observed collapse, defibrillation, and absence of vasopressor administration (multivariate analysis). Furthermore, $74.2 \%$ of survivors described their health status as good.

Interpretation: Overall, few patients survive out-of-hospital cardiac arrest in the long term. Long-term survivors rate their health status as good and show a good neurological outcome. This treatment outcome seems to depend on the same parameters as the primary resuscitation success (survival to hospital admission). The further establishment of clinical tools for early prognostication is desirable. For this purpose, further long-term studies of larger patient collectives with access to routine data are necessary.

\section{Keywords}

Cardiac arrest · Out-of-hospital cardiac arrest · Long-term outcome · Resuscitation

https://doi.org/10.1016/j.resuscitation.2019.02. 039

10. Gräsner J-T, Lefering R, Koster RW et al (2016) EuReCa ONE27 Nations, ONEEurope, ONE Registry. Resuscitation 105:188-195. https://doi.org/10. 1016/j.resuscitation.2016.06.004

11. Gräsner J-T, Wnent J, Herlitz J et al (2020) Survival after out-of-hospital cardiac arrest in Europe-Results of the Eureca TWO study. Resuscitation 148:218-226. https://doi.org/10. 1016/j.resuscitation.2019.12.042

12. Günther A, Schildmann J, in der Schmitten J et al (2020) Opportunities and risks of resuscitation attempts in nursing homes: facts for nursing home residents and caregivers. Dtsch Aerztebl Online. https://doi.org/10.3238/arztebl.2020.0757

13. Hawkes C, Booth S, Ji C et al (2017) Epidemiology and outcomes from out-of-hospital cardiac arrests in England. Resuscitation 110:133-140. https:// doi.org/10.1016/j.resuscitation.2016.10.030

14. Heckman JJ (1979) Sample selection bias as a specification error. Econometrica 47:153-161. https://doi.org/10.2307/1912352

15. Jennett B, Bond M (1975) Assessment of outcome after severe brain damage. Lancet 1:480-484. https://doi.org/10.1016/s0140-6736(75)92830-5

16. Kragholm K, Wissenberg M, Mortensen RN et al (2017) Bystander efforts and 1-year outcomes in out-of-hospital cardiac arrest. N Engl J Med 376:1737-1747. https://doi.org/10.1056/ NEJMoa1601891
17. Laver S, Farrow C, Turner D, Nolan J (2004) Mode of death after admission to an intensive care unit following cardiac arrest. Intensive Care Med 30:2126-2128. https://doi.org/10.1007/s00134004-2425-z

18. Lemiale V, Dumas F, Mongardon N et al (2013) Intensive care unit mortality after cardiac arrest: the relative contribution of shock and brain injury in a large cohort. Intensive Care Med 39:1972-1980. https://doi.org/10.1007/s00134013-3043-4

19. Müller-Nordhorn J, Roll S, Willich SN (2004) Comparison of the short form (SF)-12 health status instrument with the SF-36 in patients with coronary heart disease. Heart 90:523-527. https:// doi.org/10.1136/hrt.2003.013995

20. Nolan JP, Soar J, Cariou A et al (2015) Postreanimationsbehandlung: Kapitel 5 der Leitlinien zur Reanimation 2015 des European Resuscitation Council. Notfall Rettungsmed 18:904-931.https:// doi.org/10.1007/s10049-015-0094-9

21. Nolan JP, Soar J et al (2010) Kurzdarstellung: Sektion 1 der Leitlinien zur Reanimation 2010 des European Resuscitation Council. Notfall Rettungsmed 13:515-522. https://doi.org/10. 1007/s10049-010-1367-y

22. Perkins GD, Jacobs IG, Nadkarni VM et al (2015) Cardiac arrest and cardiopulmonary resuscitation outcome reports: update of the Utstein resuscitation registry templates for out-of-hospital cardiac arrest: a statement for healthcare professionals from a Task Force of the International Liaison 


\section{Originalien}

Committee on Resuscitation (American Heart Association, European Resuscitation Council, Australian and New Zealand Council on Resuscitation, Heart and Stroke Foundation of Canada, InterAmerican Heart Foundation, Resuscitation Council of Southern Africa, Resuscitation Council of Asia); and the American Heart Association Emergency Cardiovascular Care Committee and the Council on Cardiopulmonary, Critical Care, Perioperative and Resuscitation. Circulation 132:1286-1300. https:// doi.org/10.1161/CIR.0000000000000144

23. Perkins GD, Ji C, Deakin CD et al (2018) A randomized trial of epinephrine in out-of-hospital cardiac arrest. N Engl J Med 379:711-721. https:// doi.org/10.1056/NEJMoa1806842

24. Sasson C, Rogers MAM, Dahl J, Kellermann AL (2010) Predictors of survival from out-of-hospital cardiac arrest: a systematic review and meta-analysis. Circ Cardiovasc Qual Outcomes 3:63-81. https://doi.org/10.1161/CIRCOUTCOMES.109. 889576

25. Seewald S, Wnent J, Lefering Ret al (2019) CaRdiac Arrest Survival Score (CRASS) - A tool to predict good neurological outcome after out-of-hospital cardiac arrest. Resuscitation. https://doi.org/10. 1016/j.resuscitation.2019.10.036

26. Smith K, Andrew E, Lijovic M et al (2015) Quality of life and functional outcomes 12 months after outof-hospital cardiacarrest. Circulation 131:174-181. https://doi.org/10.1161/CIRCULATIONAHA.114. 011200

27. Sondergaard KB, Wissenberg M, Gerds TA et al (2019) Bystander cardiopulmonary resuscitation and long-term outcomes in out-of-hospital cardiac arrest according to location of arrest. Eur Heart J 40:309-318. https://doi.org/10.1093/eurheartj/ ehy687

28. Tanaka Y, Taniguchi J, Wato $Y$ et al (2012) The continuous quality improvement project for telephone-assisted instruction of cardiopulmonary resuscitation increased the incidence of bystander CPR and improved the outcomes of out-of-hospital cardiac arrests. Resuscitation 83:1235-1241. https://doi.org/10.1016/j.resuscitation.2012.02. 013

29. Ware J, Kosinski M, Keller SD (1996) A 12-Item Short-Form Health Survey: construction of scales and preliminary tests of reliability and validity. Med Care 34:220-233. https://doi.org/10.1097/ 00005650-199603000-00003

30. The Brain Resuscitation Clinical Trial II Study Group (1991) A randomized clinical trial of calcium entry blocker administration to comatose survivors of cardiac arrest. Control Clin Trials 12:525-545. https://doi.org/10.1016/0197-2456(91)90011-A 